\title{
A phase I study of sorafenib in combination with S-1 plus cisplatin in patients with advanced gastric cancer
}

\author{
Yasuhide Yamada $\cdot$ Naomi Kiyota $\cdot$ Nozomu Fuse $\cdot$ \\ Ken Kato $\cdot$ Hironobu Minami $\cdot$ Kensei Hashizume $\cdot$ \\ Yoshihiro Kuroki $\cdot$ Yuichiro Ito $\cdot$ Atsushi Ohtsu
}

Received: 15 October 2012/ Accepted: 11 February 2013/Published online: 27 March 2013

(c) The International Gastric Cancer Association and The Japanese Gastric Cancer Association 2013

\begin{abstract}
Background Sorafenib inhibits several receptor tyrosine kinases involved in tumor progression and angiogenesis. $\mathrm{S}-1$, an oral fluorouracil antitumor drug, plus cisplatin (CDDP) is the standard regimen for advanced gastric adenocarcinoma (AGC) in Japan. The purpose of this phase I study was to evaluate the safety, pharmacokinetics, and preliminary efficacy of sorafenib in combination with S-1 plus CDDP.

Methods Patients with histologically confirmed previously untreated AGC were evaluated for eligibility and treated with sorafenib (400 $\mathrm{mg}$ bid, days $1-35), \mathrm{S}-1$ (40 $\mathrm{mg} / \mathrm{m}^{2}$ bid, days $\left.1-21\right)$, and CDDP $\left(60 \mathrm{mg} / \mathrm{m}^{2}\right.$, day 8$)$. Treatment was continued until disease progression or unacceptable toxicity. Pharmacokinetics for sorafenib, 5-FU, and CDDP were investigated in cycle 1.
\end{abstract}

\author{
Y. Yamada $(\bowtie)$ \\ Gastrointestinal Medical Oncology Division, \\ National Cancer Center Hospital, 5-1-1 Tsukiji, Chuo-ku, \\ Tokyo 104-0045, Japan \\ e-mail: yayamada@ncc.go.jp \\ N. Kiyota $\cdot$ H. Minami \\ Kobe University Hospital, Hyogo, Japan \\ N. Fuse \\ National Cancer Hospital East, Chiba, Japan \\ K. Kato \\ National Cancer Center Hospital, Tokyo, Japan \\ K. Hashizume · Y. Kuroki · Y. Ito \\ Bayer Yakuhin Ltd, Osaka, Japan \\ A. Ohtsu \\ National Cancer Hospital, Chiba, Japan
}

Results Thirteen patients were enrolled and received at least one dose of the study treatment. No specific or serious adverse event was newly reported in this study. Five patients had partial response and 8 had stable disease as the best response. Pharmacokinetic analysis showed no significant differences in the exposures of sorafenib when administered alone or in combination with S- 1 and CDDP. Conclusions The present phase I study demonstrates the acceptable toxicity and preliminary efficacy of combined treatment with S-1, CDDP, and sorafenib.

Keywords Sorafenib · Phase I - S-1 · Cisplatin · Gastric adenocarcinoma

\section{Introduction}

Gastric cancer is the second leading cause of cancer death worldwide [1]. The incidence of gastric cancer has decreased during the past two decades, but the mortality rate still ranks second among all cancer types in Japan (Vital Statistics Japan, Ministry of Health, Labour and Welfare, 2009, http://ganjoho.ncc.go.jp/public/statistics/pub/ statistics02.html). Although clinical outcomes for gastric cancer have improved, the overall prognosis remains poor, especially in patients with locally advanced or metastatic disease.

Currently, the standard chemotherapeutic regimen for advanced gastric cancer in Japan is a combination of fluoropyrimidines plus platinum. S-1 is a fourth generation of oral fluoropyrimidine composed of tegafur, 5-chloro-2,4dihydroxypyridine (CDHP), and potassium oxonate (Oxo) in 1:0.4:1 molar ratio. S-1 is widely used for the treatment of gastric cancer in Japan and an increasing number of clinical trials have been conducted using this drug. A randomized 
phase III study comparing S-1 alone with S-1 plus cisplatin (CDDP) as a first-line treatment showed a significant improvement in the median survival time of patients receiving a combination of S-1 and CDDP (The SPIRITS trial [2]). More recently, a study conducted in China [3] demonstrated similar results to the SPIRITS trial. These clinical trials highlight the importance of S-1 plus CDDP as a first-line chemotherapeutic regimen for gastric cancer. However, the benefit of this new combination regimen is still modest, with a median survival time of approximately 13 months. To further improve the overall prognosis of gastric cancer patients, the development of a new combination regimen is desperately needed.

Recent advances in molecular biology have led to a better understanding of differences between cancer and normal cells at a genetic/molecular level and subsequently to the development of novel therapeutic strategies to specifically block the molecules required for cancer growth and metastasis. So far, many preclinical studies and clinical trials have been conducted using molecular-targeting agents such as epidermal growth factor receptor (EGFR)-targeting agents and vascular endothelial growth factor (VEGF)-targeting agents. Sorafenib (BAY 43-9006), identified through a screening for Raf kinase inhibitors, is a potent multikinase inhibitor and one of the promising targeted drugs currently used in the clinical setting. Antitumor efficacy of sorafenib depends on potent inhibition of c-Raf and wildtype and mutant-b-Raf, combined with inhibition of mitogen-activated protein (MAP) kinase signaling pathway and of tumor angiogenesis via vascular endothelial growth factor receptor-2 (VEGFR-2) and platelet-derived growth factor receptor- $\beta$ (PDGFR- $\beta$ ) [4]. Sorafenib has been approved in many countries for the treatment of advanced renal cell carcinoma and hepatocellular carcinoma.

Activation of the MAP kinase signaling pathway via EGF/EGFR signaling has been reported in human gastric cancer. Moreover, tumor angiogenesis through the VEGF/ VEGFR signaling is involved in the progression of gastric cancer [5, 6]. Therefore, these signaling pathways could be candidate targets for molecular-targeted therapy against gastric cancer. Boku et al. [7] examined the correlation between survival and VEGF expression in patients with gastric cancer treated with S-1 or S-1 plus CDDP. Although the survival of patients with VEGF-negative tumors was slightly longer than those with VEGF-positive tumors in a group treated with S-1 alone, patients with VEGF-positive tumors survived remarkably longer than those with VEGF-negative tumors in a group treated with a combination of S- 1 and CDDP. The authors suggested that clinical benefit of adding CDDP to S-1 might be more pronounced in patients with VEGF-positive tumors than in those with VEGF-negative tumors [7]. Another study has shown that EGFR expression correlates with worse prognosis in gastric cancer patients [8]. These findings together suggest the potential efficacy of sorafenib for the treatment of gastric cancer. In fact, the combination of sorafenib, docetaxel, and cisplatin was investigated in a phase II study for the treatment of metastatic or advanced gastric cancer and showed promising results [9].

Addition of sorafenib to the standard regimen of S-1 plus CDDP could enhance the therapeutic efficacy in an additive or synergistic manner and further improve the survival rate of patients with gastric cancer. We therefore conducted this phase I study, before a large-scale controlled trial, to investigate the safety, pharmacokinetics, and pharmacodynamics of sorafenib in combination with S-1 plus CDDP for patients with unresectable or recurrent gastric cancer.

\section{Patients and methods}

\section{Patient eligibility}

The main inclusion criteria were as follows: patients with histologically or cytologically confirmed unresectable or recurrent gastric adenocarcinoma; age between 18 and 74 years; no prior chemotherapy or immunotherapy; Eastern Cooperative Oncology Group performance status (ECOG-PS) of $0-1$; an estimated life expectancy of $\geq 12$ weeks; and adequate bone marrow, liver, and renal function within 7 days before dosing [hemoglobin $\geq 8.5 \mathrm{~g} / \mathrm{dl}$, absolute neutrophil count (ANC) $\geq 1,500 / \mathrm{mm}^{3}$, platelet count $\geq 100,000 / \mathrm{mm}^{3}$, total bilirubin $\leq 1.5 \times$ upper limit of normal (ULN), alanine aminotransferase (ALT) and aspartate aminotransferase (AST) $\leq 2.5 \times$ ULN, creatinine clearance $\geq 60 \mathrm{ml} / \mathrm{min}$ ). Prior adjuvant therapy/neo-adjuvant therapy is allowed if recurrence occurred 6 months after completion of these therapies. The main exclusion criteria were as follows: patients with brain metastasis; ascites; active bacterial infection; fungal infection; chronic hepatitis $\mathrm{B} / \mathrm{C}$; pregnancy; and hemodialysis.

The institutional review board approval was obtained at each participating institution. The study was performed in accordance with the Declaration of Helsinki and Good Clinical Practice Guidelines. All patients gave written informed consent after receiving a sufficient explanation before study treatment.

\section{Study design}

This is a multicenter (Gastrointestinal Oncology Division, National Cancer Center Hospital, Tokyo, Japan; Division of Gastrointestinal Oncology, National Cancer Center Hospital East, Chiba, Japan; Division of Medical Oncology, Kobe University Hospital, Hyogo, Japan), unblinded, 
uncontrolled phase I trial. The primary objective is to investigate the safety and pharmacokinetics of sorafenib administered in combination with $\mathrm{S}-1$ plus CDDP to patients with unresectable or recurrent gastric cancer (chemotherapy-naive for advanced disease). The secondary objective is to investigate preliminary efficacy profile.

The phase III trials, the SPIRITS and the FLAGS studies, using S-1 plus CDDP for advanced gastric cancerbased regimen, were referred to for the study design. Initially three cohorts were planned for this study. Treatment schedule and dosage were set based on the SPIRITS study regimen for cohort 1 and on the FLAGS study regimen for cohorts 2 and 3. Cohorts 2 and 3 were canceled because of negative results of the FLAGS study reported during conduct of cohort 1 [10].

The dosages of S-1 and CDDP in cohort 1 were based on the SPIRITS study as follows: $\mathrm{S}-1,40 \mathrm{mg} / \mathrm{m}^{2}$, p.o. twice daily 21 days followed by 14 days rest; CDDP, $60 \mathrm{mg} / \mathrm{m}^{2}$, i.v. on day 8. Sorafenib was continuously administered orally at $400 \mathrm{mg}$ twice a day. Each cycle was defined as 35 days. In terms of cycle 1, sorafenib was orally administered continuously on days 2-35 for pharmacokinetics evaluation (Fig. 1). In this study, strict dose reduction criteria in a two-step process were defined for each drug. Dose reduction or discontinuation of the drug was determined according to the grades of the adverse events developed during study treatment. The dose reduction levels of S-1 included level 1 (20\% reduction) and level 2 (35\% reduction). The dose reduction levels of CDDP included level 1 (25\% reduction) and level 2 (50\% reduction). The dose reduction levels of sorafenib included level 1 (50\% reduction, $400 \mathrm{mg}$ once daily) and level 2 (75\% reduction, $400 \mathrm{mg}$ once every second day).

\section{Pharmacokinetics (PK)}

Patients who have at least one evaluable PK profile were valid for PK analysis. Blood samples (5-ml aliquots) for the determination of plasma concentration of sorafenib were collected at $0 \mathrm{~h}$ (pre-morning dose), and 1, 2, 4, and $8 \mathrm{~h}$ after morning dose, and before evening dose $(\sim 12 \mathrm{~h}$ after morning dose) on days 8 and 35 in cycle 1 .

Blood samples (10-ml aliquots) for the determination of plasma concentration of 5-FU (for S-1) were collected at $0 \mathrm{~h}$ (pre-morning dose), and 1, 2, 4, and $8 \mathrm{~h}$ after morning dose and before evening dose ( $\sim 12 \mathrm{~h}$ after morning dose) on days 1,8 , and 15 in cycle 1 .

Blood samples (6-ml aliquots) for the determination of plasma concentration of total and free platinum were collected at $0 \mathrm{~h}$ (pre-dose), end of infusion, and $2 \mathrm{~h}$ after end of infusion on day 8 in cycle 1 , at $24 \mathrm{~h}$ after starting of infusion, and at any three time points among 48, 72, 96, 120 , and $144 \mathrm{~h}$ after starting of infusion.

Plasma concentrations of sorafenib and 5-FU were determined by validated liquid chromatography-tandem mass spectrometry (LC/MS/MS) after protein precipitation.

Plasma platinum concentrations were measured by quantifying total and free platinum by validated flameless atomic absorption spectrometry (FAAS) after dilution [11]. Depending on the concentration of the sample, a certain volume of Triton-X solution (1\%) or nitric acid (6.5\%) was added. For analysis of free platinum in plasma, plasma samples were centrifuged with filter devices (Millipore, Centrifree YM-30) to obtain an ultrafiltrate fraction.

The pharmacokinetic parameters of sorafenib, total and free platinum, and 5-FU were calculated using the modelindependent (non-compartment) method and the PC program WinNonlin.

Pharmacodynamics (PD)

\section{Plasma protein biomarkers}

To measure protein levels in plasma, enzyme-linked immunosorbent assay (ELISA) kits for EGFR (Siemens Healthcare Diagnostics, Tarrytown, NY, USA), HER2/neu (Siemens Healthcare Diagnostics), PDGFR- $\beta$ (R\&D Systems, Minneapolis, MN, USA), VEGF (detecting VEGF-

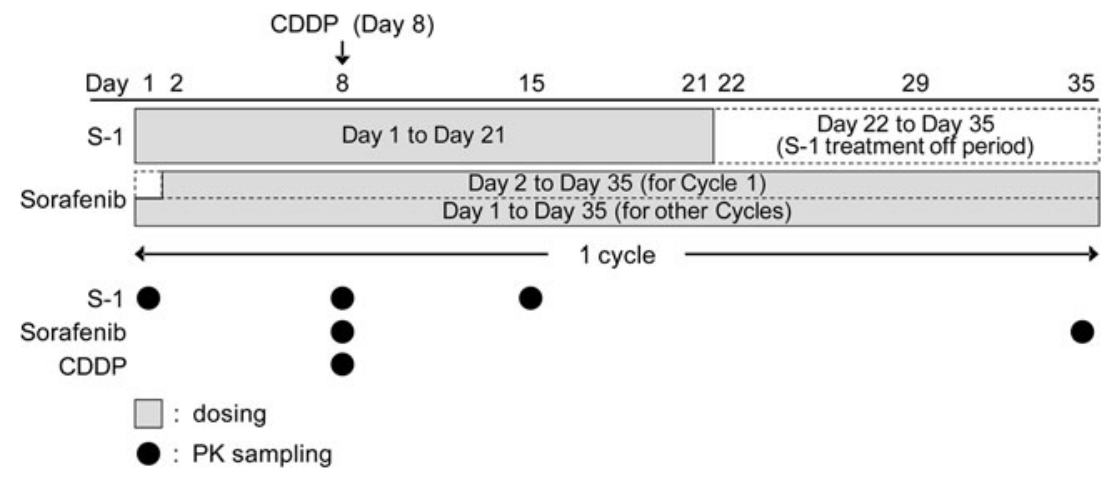

Fig. 1 Treatment cycle [drug administration and pharmacokinetics (PK) sampling schedule] 
165; R\&D Systems), and sVEGFR-2 (R\&D Systems) were used according to the manufacturers' specifications.

\section{Immunohistochemical (IHC) analysis}

The anti-VEGFR2 rabbit monoclonal antibody (mAb) (clone 55B11), the anti-AKT rabbit mAb (clone C67E7), the anti-phosphorylated AKT (pAKT) rabbit mAb (clone D9E), the anti-ERK mouse mAb (clone 3A7), and the antiphosphorylated ERK (pERK) rabbit mAb (clone D13.14.4E) were purchased from Cell Signaling Technology (Danvers, MA, USA). The anti-pAKT rabbit mAb (clone 14-5), was purchased from Dako (Carpinteria, CA, USA). The anti-pERK mouse mAb (clone MAPK-YT) was purchased from Sigma (St. Louis, MO, USA). The mouse IgG isotype control antibody and the rabbit IgG isotype control antibody were purchased from Dako. For AKT, pAKT, ERK, and pERK, the staining intensity of each section was determined by comparing the intensity of a control slide containing an adjacent section stained with an irrelevant, negative control antibody that is species- and isotype matched to the test article. The staining intensity was classified as follows: 0 , no staining relative to background; $1+$, weak staining; $2+$, moderate staining; and 3+, strong staining. The $\mathrm{H}$-score was calculated based on the summation of the product of percent of cells stained at each intensity using the following equation: $(3 \times \%$ cells staining at $3+)+(2 \times \%$ cells staining at $2+)+(1 \times \%$ cells staining at $1+$ ). Evaluation of VEGFR2 was performed by pixel analysis. Stained slides were scanned on an Aperio CS Digital Slide Scanner (Vista, CA, USA), and results were evaluated using the Positive Pixel V9 algorithm. The fraction of positive to total stained pixels was determined and reported as the percent positive for each staining threshold.

\section{Microarray analysis of biopsy specimens}

RNA was isolated from endoscopic biopsy specimens according to the Qiagen RNeasy Micro Handbook (Qiagen, Hilden, Germany, 2007). An aliquot (100 ng) of total RNA from each biopsy specimen was first reverse transcribed using a T7-Oligo(dT) Promoter Primer in the first-strand cDNA synthesis reaction followed by a RNase H-mediated second-strand cDNA synthesis. The subsequent in vitro transcription (IVT) reaction was carried out in the presence of T7 RNA polymerase and a biotinylated nucleotide analogue/ribonucleotide mix for amplified RNA (aRNA) amplification and biotin labeling. The labeled aRNA was fragmented and hybridized to an oligonucleotide microarray HG-U133 Plus 2.0 array (Affymetrix, Santa Clara, CA, USA). The arrays were washed and stained using the GeneChip Fluidics Station 450, then scanned by
GeneChip-3000 Scanner at $570 \mathrm{~nm}$. All reactions were performed using the 3'IVT Express Labeling and Control Reagents from Affymetrix. Data were analyzed using the Affymetrix MAS 5.0 algorithm. Tests for differential expression were performed for each transcript separately and did not take dependencies between transcripts into account. The transcripts were then sorted by the respective measures of statistical significance ( $p$ values). Adjustments for multiple hypothesis testing were performed through the use of the false discovery rate (FDR). Statistical analysis was performed by means of the SAS System Version 9.1.3 (SAS Institute, Cary, NC, USA) and the Bioconductor packages [12] from release 2.7 within $\mathrm{R}$ software version 2.12.1. A gene ontology (GO) classification of the differentially expressed genes with corrected $p$ values $<0.05$ was carried out by the GO Term Finder [13].

\section{Clinical assessment}

Physical examination, complete blood cell counts, serum chemistries, and urinalysis were carried out at baseline and at least every week for the first two cycles after initiating treatment and three times per cycle during cycle 3 and 4, two times per cycle from cycle 5. All observations on the safety of the treatment were recorded, and patients were routinely monitored for adverse events, which were recorded with severity and relationship to study medication according to the National Cancer Institute (NCI) Common Terminology Criteria for Adverse Events (CTCAE) version 3.0. Tumor response was assessed every 8 weeks for the first six cycles and every 12 weeks after cycle 6 by Response Evaluation Criteria in Solid Tumors (RECIST) version 1.0 using the same imaging techniques and methods used at baseline.

\section{Results}

Patient characteristics

From May 2008 to January 2009, a total of 13 chemo-naive patients (10 male and 3 female patients with a mean age of 61 years) with advanced gastric cancer were enrolled in this study (Table 1).

Treatment compliance and duration

The median number of treatment cycles was 4 (range 1-12 cycles). Of 13 patients, 8 were able to continue at least 4 cycles of treatment, and 6 of them had received 5 cycles or more. The median relative dose intensity (RDI) of sorafenib $(50.0 \%)$ was lower than those of S-1 $(89.3 \%)$ or CDDP (92.0\%) (Table 2). Focusing on median RDI from 
cycle 1 to 4, RDI of sorafenib was gradually decreasing, whereas those of S-1 and CDDP were sustained around $80 \%$ (Table 3 ); this could be partly because dose reduction criteria were different between these drugs $(50 \%$ reduction for sorafenib versus $20 \%$ for S-1 and $25 \%$ for CDDP at level 1). Relatively large numbers of patients required dose reduction or interruption because of adverse events of sorafenib or S-1 (Table 4). Seven of 13 patients discontinued study treatment permanently for reasons of adverse events such as diarrhea, elevation of transaminase,

Table 1 Baseline demographics and disease characteristics

\begin{tabular}{|c|c|c|c|}
\hline$n=13$ & $n(\%)$ & & $n(\%)$ \\
\hline \multicolumn{2}{|l|}{ Sex } & \multicolumn{2}{|l|}{ Histological type } \\
\hline Male & $10(77)$ & Adenocarcinoma & $13(100)$ \\
\hline Female & $3(23)$ & (tub:por:sig:muc) & $(6: 5: 1: 1)$ \\
\hline \multicolumn{2}{|l|}{ Age (years) } & \multicolumn{2}{|c|}{ Sites of metastatic lesion } \\
\hline Median (range) & $61(41-72)$ & Lymph nodes & $10(77)$ \\
\hline \multicolumn{2}{|c|}{ ECOG performance status } & Liver & $7(54)$ \\
\hline 0 & $9(69)$ & Peritoneum & $1(8)$ \\
\hline 1 & $4(31)$ & Lung & $1(8)$ \\
\hline \multicolumn{2}{|l|}{ Prior gastrectomy } & Bone & $1(8)$ \\
\hline Yes & $3(23)^{\mathrm{a}}$ & & \\
\hline No & $10(77)$ & & \\
\hline \multicolumn{4}{|c|}{ Prior systemic anticancer therapy } \\
\hline Yes & $0(0)$ & & \\
\hline No & $13(100)$ & & \\
\hline
\end{tabular}

ECOG Eastern Cooperative Oncology Group, tub tubular adenocarcinoma, por poorly differentiated adenocarcinoma, sig signet ring cell carcinoma, тис mucinous adenocarcinoma

${ }^{a}$ Distal gastrectomy

Table 2 Received dose intensity (\%) of each drug

\begin{tabular}{llll}
\hline & $\begin{array}{l}\text { Sorafenib }(\%) \\
(n=13)\end{array}$ & $\begin{array}{l}\text { S-1 }(\%) \\
(n=13)\end{array}$ & $\begin{array}{l}\text { CDDP }(\%) \\
(n=13)\end{array}$ \\
\hline $\begin{array}{c}\text { Mean } \\
(\mathrm{SD})\end{array}$ & $53.9(18.3)$ & $85.2(13.1)$ & $86.2(16.7)$ \\
$\begin{array}{c}\text { Median } \\
\text { (range) }\end{array}$ & $50.0(31.8-96.0)$ & $89.3(55.0-100.0)$ & $92.0(50.0-100.0)$ \\
\hline
\end{tabular}

$S D$ standard deviation myelosuppression, and hand-foot skin reaction (Table 5). The other 6 patients discontinued treatment permanently because of progressive disease (PD).

\section{Safety}

The most common adverse events were anorexia (100\%), rash/desquamation (100\%), neutropenia (92\%), thrombocytopenia (92\%), hand-foot reaction and nausea (85\%), leukopenia, fatigue, and elevation of lipase (77\%) (Table 6). Seven (54\%) patients discontinued study treatment because of adverse events such as diarrhea (2 patients), anemia, neutropenia, gastric perforation, hand-foot skin reaction, transaminase increase (1 patient, respectively). Although the ratio of skin rash was high (100\%), all were relatively mild (grades 1 and 2), and only a few patients required dermatologist consultation. No treatment-related death was observed during the study period.

\section{Efficacy}

Tumor response was evaluated in 12 patients having a target lesion for assessment according to RECIST 1.0 criteria by the investigator. The overall response rate was $38.5 \%$ [95\% confidence interval (CI), 13.9-68.4] (Table 7). Remarkably, all the 12 patients evaluable for tumor response showed reduction of target lesion after study treatment compared with baseline on one level or another (Fig. 2).

The median change in percentage of target lesions was $-22.9 \%$ (range -8.9 to $-100 \%$ ). In 1 patient, the target lesion was shrunk by $100 \%$ after study treatment, whereas all the non-target legions did not show CR. Therefore, this patient was regarded as PR overall.

One-year survival rate was $76.9 \%$ (95\% CI, $44.2 \%$, $91.9 \%)$.

Pharmacokinetics (PK)

\section{Sorafenib}

Plasma concentration-time profiles of sorafenib were available in 4 patients on day 35 (sorafenib alone) and in 11

Table 3 Relative dose intensity (RDI) of each drug at each treatment cycle

\begin{tabular}{lllllll}
\hline & & Sorafenib (\%) & & S-1 (\%) & \multicolumn{2}{c}{ CDDP (\%) } \\
\hline Cycle 1 & $n=13$ & $77.8(41.2-100.0)$ & $n=13$ & $100.0(61.9-100.0)$ & $n=13$ & $100.0(100.0-100.0)$ \\
Cycle 2 & $n=11$ & $46.4(28.1-100.0)$ & $n=11$ & $81.0(45.8-100.0)$ & $n=10$ & $100.0(0.0-100.0)$ \\
Cycle 3 & $n=8$ & $47.2(25.0-100.0)$ & $n=8$ & $83.3(66.7-100.0)$ & $n=8$ & $75.0(0.0-100.0)$ \\
Cycle 4 & $n=8$ & $36.0(25.0-74.3)$ & $n=8$ & $83.3(56.9-100.0)$ & $n=7$ & $75.0(75.0-100.0)$ \\
\hline
\end{tabular}

Values are expressed as median (range) 
Table 4 Number of patients who required dose reduction or interruption because of adverse events from each drug

\begin{tabular}{lcrl}
\hline & Sorafenib & \multicolumn{1}{l}{ S-1 } & CDDP \\
\hline \multicolumn{2}{l}{ Number of subjects with dose reduction } \\
No & $1(8 \%)$ & $3(23 \%)$ & $8(62 \%)$ \\
Yes & $12(92 \%)$ & $10(77 \%)$ & $5(38 \%)$ \\
Number of subjects with dose interruption & \\
No & 0 & $5(38 \%)$ & $9(69 \%)$ \\
Yes & $13(100 \%)$ & $8(62 \%)$ & $4(31 \%)$ \\
\hline
\end{tabular}

Efficacy analysis set, $n=13$

Table 5 Adverse events leading to discontinuation of study drug in seven patients

\begin{tabular}{lll}
\hline Case number & Adverse event & End of cycle \\
\hline 1 & AST & Cycle 1 \\
& ALT & \\
2 & Diarrhea & Cycle 2 \\
& Mucositis & \\
3 & Perforation, GI, Stomach & Cycle 2 \\
4 & Hypophosphatemia & Cycle 1 \\
& Diarrhea & \\
5 & Hemoglobin & Cycle 6 \\
7 & Hand-foot skin reaction & Cycle 2 \\
\hline
\end{tabular}

patients on day 8 (in combination of S-1 plus CDDP). There appeared to be no difference in the geometric mean plasma concentration profiles of sorafenib between in patients receiving sorafenib alone and those receiving a combination of three drugs (Fig. 3).

We evaluated geometric means [\% coefficient of variation $(\mathrm{CV})] C_{\max }$ and $\mathrm{AUC}_{0-\mathrm{tn}}$ of sorafenib in patients receiving sorafenib alone (day 35 ) and in those receiving a combined administration of sorafenib, $\mathrm{S}-1$, and CDDP (day 8), as well as the mean ratios of $C_{\max }$ and $\mathrm{AUC}_{0-\text { tn }}$ of sorafenib (value at combination treatment on day 8/value at sorafenib monotherapy on day 35) in four patients obtained on both day 8 and day 35 (Table 8 ). These pharmacokinetic data showed no significant change in $\mathrm{AUC}_{0-\mathrm{tn}}$ and $C_{\max }$ of sorafenib under a combined administration of $\mathrm{S}-1$ and CDDP.

\section{$5-F U$}

Plasma concentration-time profiles of 5-FU were available in 13 patients on day 1 ( $\mathrm{S}-1$ alone) and day 8 (in combination with sorafenib and CDDP).

Geometric means of plasma 5-FU concentrations were slightly higher when 5-FU was administered in combination with the other two drugs (day 8) than that when administered alone (day 1) (Fig. 4).

Mean ratios (day 8/day 1) of $C_{\max }$ and $\mathrm{AUC}_{0-\mathrm{tn}}$ of 5-FU were 1.64 and 1.56 , respectively, in 13 patients in whom these measurements were obtained on both day 1 and day 8 (Table 9).

Both $\mathrm{AUC}_{0-\mathrm{tn}}$ and $C_{\max }$ were higher when $\mathrm{S}-1$ was administered in combination with the other drugs (day 8) than that when administered alone (day 1).

\section{$C D D P$}

Because the PK of CDDP administered alone was not examined in this study, the effect of concomitant administration of sorafenib and S-1 on the PK of CDDP could not be evaluated.

Geometric mean $(\% \mathrm{CV}) C_{\max }$ and $\mathrm{AUC}_{0-\text { tn }}$ of total platinum on day 8 were $3.06 \mathrm{mg} / \mathrm{l} \quad(14.5 \%)$ and $152.28 \mathrm{mg} \cdot \mathrm{h} / \mathrm{l}(14.8 \%)$, respectively (Table 10$)$. Geometric mean $(\% \mathrm{CV}) C_{\max }$ and $\mathrm{AUC}_{0-\mathrm{tn}}$ of free platinum on day 8 was $1.25 \mathrm{mg} / \mathrm{l}(15.8 \%)$ and $4.47 \mathrm{mg} \cdot \mathrm{h} / \mathrm{l}(39.6 \%)$, respectively (Table 10). $T_{\max }$ was $1.98 \mathrm{~h}$ for both total platinum and free platinum.

\section{Pharmacodynamics (PD)}

Blood samples were taken from 12 patients during screening and on day 15 of cycle 1 . Changes in plasma concentrations of biomarkers, including VEGFR2, VEGF, PDGFR- $\beta$, EGFR, and HER2/neu, were plotted individually and compared before and during treatment (day 15) (Fig. 5). Among these markers, plasma concentration of EGFR tended to increase more during treatment in patients showing PR [median percent change from screening, $55.9 \%(n=4)$ ] than in those showing SD [median percent change from screening, $-2.0 \% \quad(n=8)]$ classified according to RECIST.

For IHC analysis, six patients giving his/her consent provided two endoscopic biopsy specimens taken at a screening period and during treatment (at the end of cycle 2). In total, 24 formalin-fixed, paraffin-embedded (FFPE) tumor specimens were analyzed. After review of hematoxylin and eosin (H\&E)-stained slides, 12 specimens (6 pairs of specimens obtained at a screening period and during treatment) were selected and stained for VEGFR2, AKT, pAKT, ERK, and pERK. IHC analyses for pAKT and pERK were done with two mAbs because both clones indicated adequate specificity and sensitivity for IHC assays in Western blot analysis and FFPE cell pellet IHC experiments (data not shown). Decrease in pAKT staining during treatment was shown by two different antibodies in patient 20001-1003 while total AKT increased. However, change in pAKT was not obvious in another patient. Also, a slight 
Table 6 Incidence of adverse events by NCI CTCAE version 3.0

\begin{tabular}{|c|c|c|c|c|c|c|}
\hline \multirow[t]{3}{*}{$\mathrm{AE}$ term } & \multicolumn{6}{|c|}{$n=13$} \\
\hline & \multicolumn{6}{|c|}{ Grade } \\
\hline & 1 & 2 & 3 & 4 & All & $\geq 3$ \\
\hline \multicolumn{7}{|l|}{ Hematological } \\
\hline Neutropenia & 0 & 5 & 4 & 3 & $12(92 \%)$ & $7(54 \%)$ \\
\hline Thrombocytopenia & 5 & 4 & 3 & 0 & $12(92 \%)$ & $3(23 \%)$ \\
\hline Leukocytopenia & 2 & 5 & 2 & 1 & $10(77 \%)$ & $3(23 \%)$ \\
\hline Anemia & 0 & 4 & 3 & 0 & $7(54 \%)$ & $3(23 \%)$ \\
\hline Lymphopenia & 0 & 2 & 1 & 0 & $3(23 \%)$ & $1(8 \%)$ \\
\hline \multicolumn{7}{|l|}{ Nonhematological } \\
\hline Anorexia & 7 & 4 & 2 & 0 & $13(100 \%)$ & $2(15 \%)$ \\
\hline Rash/desquamation & 9 & 4 & 0 & 0 & $13(100 \%)$ & 0 \\
\hline Hand-foot skin reaction & 2 & 6 & 3 & 0 & $11(85 \%)$ & $3(23 \%)$ \\
\hline Metabolic/lab, other & 2 & 9 & 0 & 0 & $11(85 \%)$ & 0 \\
\hline Nausea & 8 & 2 & 1 & 0 & $11(85 \%)$ & $1(8 \%)$ \\
\hline Fatigue & 6 & 1 & 3 & 0 & $10(77 \%)$ & $3(23 \%)$ \\
\hline Lipase & 0 & 2 & 5 & 3 & $10(77 \%)$ & $8(62 \%)$ \\
\hline Alopecia & 8 & 1 & 0 & 0 & $9(69 \%)$ & 0 \\
\hline Amylase & 5 & 1 & 3 & 0 & $9(69 \%)$ & $3(23 \%)$ \\
\hline Diarrhea & 4 & 2 & 2 & 0 & $8(62 \%)$ & $2(15 \%)$ \\
\hline Mucositis (functional/symptomatic), oral cavity & 3 & 2 & 2 & 0 & $7(54 \%)$ & $2(15 \%)$ \\
\hline Vomiting & 6 & 1 & 0 & 0 & $7(54 \%)$ & 0 \\
\hline Bilirubin (hyperbilirubinemia) & 1 & 4 & 1 & 0 & $6(46 \%)$ & $1(8 \%)$ \\
\hline Constipation & 5 & 1 & 0 & 0 & $6(46 \%)$ & 0 \\
\hline Hiccoughs & 1 & 5 & 0 & 0 & $6(46 \%)$ & 0 \\
\hline AST & 2 & 2 & 0 & 1 & $5(39 \%)$ & $1(8 \%)$ \\
\hline Hypertension & 1 & 2 & 2 & 0 & $5(39 \%)$ & $2(15 \%)$ \\
\hline ALT & 3 & 0 & 0 & 1 & $4(31 \%)$ & $1(8 \%)$ \\
\hline Fever & 2 & 2 & 0 & 0 & $4(31 \%)$ & 0 \\
\hline Hyperpigmentation & 4 & 0 & 0 & 0 & $4(31 \%)$ & 0 \\
\hline Hypophosphatemia & 0 & 2 & 2 & 0 & $4(31 \%)$ & $2(15 \%)$ \\
\hline Periodontal & 3 & 1 & 0 & 0 & $4(31 \%)$ & 0 \\
\hline Taste alteration & 3 & 1 & 0 & 0 & $4(31 \%)$ & 0 \\
\hline Flu-like syndrome & 3 & 0 & 0 & 0 & $3(23 \%)$ & 0 \\
\hline Hemorrhage, other (specify) & 3 & 0 & 0 & 0 & $3(23 \%)$ & 0 \\
\hline Nail changes & 3 & 0 & 0 & 0 & $3(23 \%)$ & 0 \\
\hline Proteinuria & 2 & 1 & 0 & 0 & $3(23 \%)$ & 0 \\
\hline Weight loss & 2 & 1 & 0 & 0 & $3(23 \%)$ & 0 \\
\hline
\end{tabular}

increase in pERK was observed by two different antibodies while total ERK remained unchanged (Table 11).

For exploratory gene expression analysis, 30 endoscopic biopsy specimens were obtained from 9 patients during a screening period and during treatment (at the end of cycle 2). One sample whose scaling factor was out of range was excluded from statistical analysis. Differential expression analysis identified a total of 624 differentially regulated probe sets in response to treatment. Analysis of the error rates indicated that the differences between differentially and nondifferentially expressed probe sets were not very well resolved. Among 624 probe sets, 469 could be assigned to GO terminologies. Of the 10 transcripts showing the most significant differential expression, 7 fell under GO annotations indicating their involvement in cell structural components (cytoskeleton, cell membranes) or membrane transport processes. Another transcript (AC133561.1), although not annotated in the GO, was identified as a transmembrane transport protein (Table 12). 
Table 7 Response rate and best overall response based on investigator assessment

\begin{tabular}{ll}
\hline & $n=13$ \\
\hline Response rate (95\% CI) & $38.5 \%(13.9-68.4)$ \\
Complete response (CR) & 0 \\
Partial response (PR) & $5(38.5 \%)$ \\
Stable disease (SD) & $8(61.5 \%)$ \\
Progressive disease (PD) & 0 \\
\hline
\end{tabular}

CI confidence interval

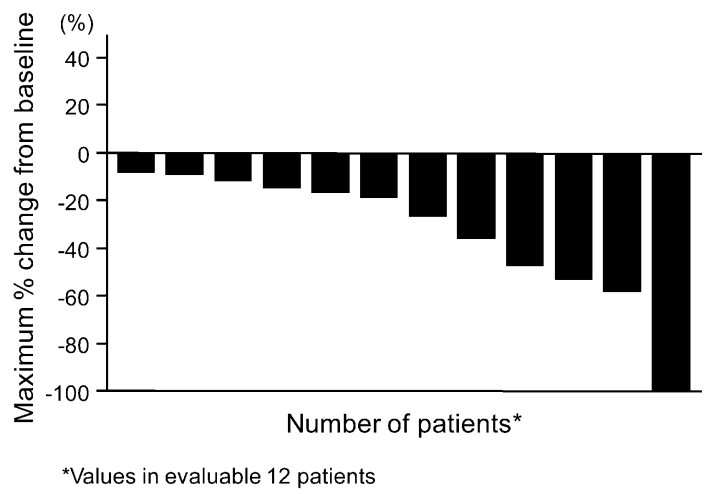

Fig. 2 Maximum percent reduction of target lesions in individual patients

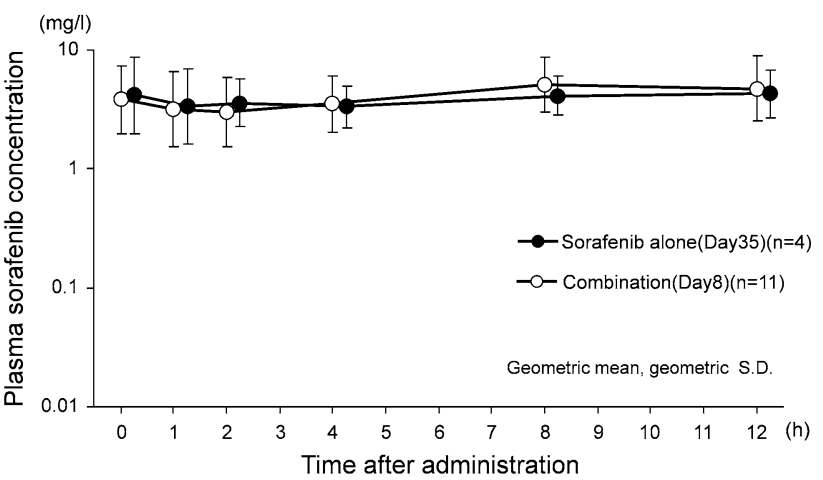

Fig. 3 Serial change in plasma concentrations of sorafenib in patients receiving sorafenib alone $(400 \mathrm{mg}$ p.o., twice a day on days 2-35) and in those receiving a combined administration of sorafenib, $\mathrm{S}-1$, and cisplatin (CDDP) $\left(\mathrm{S}-1,40 \mathrm{mg} / \mathrm{m}^{2}\right.$, p.o., twice a day for 21 days followed by 14 days rest; CDDP, $60 \mathrm{mg} / \mathrm{m}^{2}$, i.v., on day 8 ; sorafenib, $400 \mathrm{mg}$ p.o., twice a day on days $2-35$ for cycle 1 and days $1-35$ for other cycles)

\section{Discussion}

In this phase I clinical trial, we evaluated the safety, pharmacokinetics, and efficacy of sorafenib in combination with S-1 and CDDP in Japanese patients with advanced gastric cancer. This combination therapy was tolerated and demonstrated antitumor activity. Pharmacokinetic results suggested that the combination of S-1 and CDDP did not
Table 8 Pharmacokinetics parameters of sorafenib

\begin{tabular}{lllll}
\hline Sorafenib & $\begin{array}{l}\text { Day 35 } \\
\text { (alone) }\end{array}$ & $\begin{array}{l}\text { Day 8 } \\
\text { (combination) }\end{array}$ & $\begin{array}{l}\text { Combination/ } \\
\text { alone ratio }\end{array}$ \\
\hline$C_{\max }$ & $n$ & 4 & 11 & 4 \\
& $\mathrm{mg} / 1$ & $5.582(51.6)$ & $5.872(58.7)$ & $\begin{array}{l}1.02 \\
(0.62-1.66)\end{array}$ \\
$\mathrm{AUC}_{0-\mathrm{tn}}$ & $n$ & 4 & 11 & 4 \\
& $\mathrm{mg} \cdot \mathrm{h} / \mathrm{l}$ & $\begin{array}{c}38.551 \\
(39.2)\end{array}$ & $37.348(53.6)$ & $\begin{array}{l}0.90 \\
(0.59-1.39)\end{array}$ \\
\end{tabular}

Geometric mean (\% coefficient of variation)

Combination/alone ratio is given as ratio (90\% confidence interval)

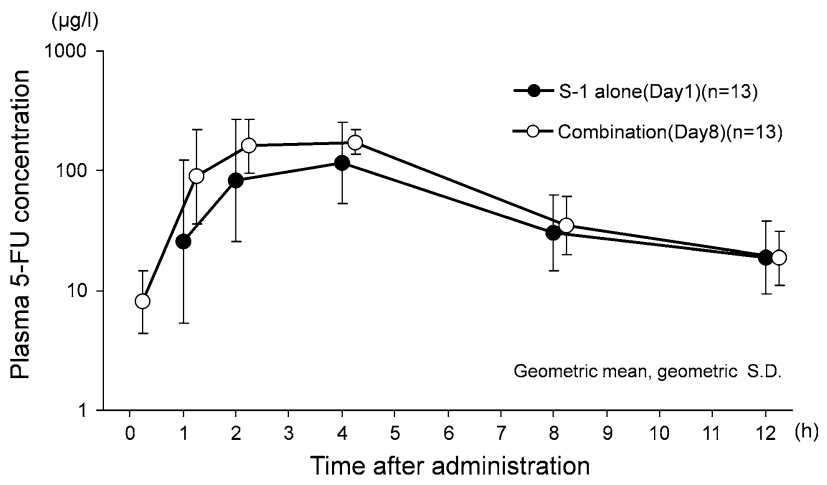

Fig. 4 Serial change in plasma concentrations of fluorouracil (5-FU) in patients receiving S-1 alone $\left(40 \mathrm{mg} / \mathrm{m}^{2}\right.$, p.o., twice a day) and in those receiving a combined administration of sorafenib, S-1, and CDDP (S-1, $40 \mathrm{mg} / \mathrm{m}^{2}$, p.o., twice a day for 21 days followed by 14 days rest; CDDP, $60 \mathrm{mg} / \mathrm{m}^{2}$, i.v., on day 8; sorafenib, $400 \mathrm{mg}$ p.o., twice a day on days 2-35 for cycle 1 and days $1-35$ for other cycles)

Table 9 Pharmacokinetics parameters of fluorouracil (5-FU)

\begin{tabular}{lllll}
\hline 5-FU & & $\begin{array}{l}\text { Day 1 } \\
\text { (alone) }\end{array}$ & $\begin{array}{l}\text { Day 8 } \\
\text { (combination) }\end{array}$ & $\begin{array}{l}\text { Combination/ } \\
\text { alone ratio }\end{array}$ \\
\hline$C_{\max }$ & $n$ & 13 & 13 & 13 \\
& $\mathrm{mg} / \mathrm{l}$ & $0.123(95.6)$ & $0.202(33.1)$ & $1.64(1.24-2.16)$ \\
$\mathrm{AUC}_{0-\mathrm{tn}}$ & $n$ & 13 & 13 & 13 \\
& $\mathrm{mg} \cdot \mathrm{h} / 1$ & $0.613(82.0)$ & $0.955(24.6)$ & $1.56(1.19-2.04)$
\end{tabular}

Geometric mean (\% coefficient of variation)

Combination/alone ratio is given as ratio (90\% confidence interval)

affect the PK of sorafenib. There was no unpredictable adverse event associated with the combination of the three drugs. Analysis of biomarkers suggested a possible link between plasma EGFR level and treatment response.

Sorafenib is a promising antitumor agent against a broad range of tumors, including hepatocellular carcinoma and renal cell carcinoma. Sorafenib has also been tested for its efficacy in gastric cancer in combination with other chemotherapeutic agents. Recently, a phase I study was 
reported testing sorafenib in combination with capecitabine and cisplatin as a first-line treatment in patients with advanced gastric cancer [14]. In addition, sorafenib in combination with docetaxel and cisplatin was tested for

Table 10 Pharmacokinetics parameters of cisplatin (CDDP) on day 8 CDDP

\begin{tabular}{llll}
\hline CDDP & & Total platinum & Free platinum \\
\hline$C_{\max }$ & $n$ & 13 & 13 \\
& $\mathrm{mg} / \mathrm{l}$ & $3.065(14.5)$ & $1.246(15.8)$ \\
$\mathrm{AUC}_{0-\mathrm{tn}}$ & $n$ & 13 & 12 \\
& $\mathrm{mg} \cdot \mathrm{h} / \mathrm{l}$ & $152.282(14.8)$ & $4.472(39.6)$ \\
$t_{\max }$ & $n$ & 13 & 13 \\
& $\mathrm{~h}$ & $1.98(1.93-2.32)$ & $1.98(1.93-2.32)$ \\
\hline
\end{tabular}

Geometric mean (\% coefficient of variation)

$t_{\max }$ is given as median (range)
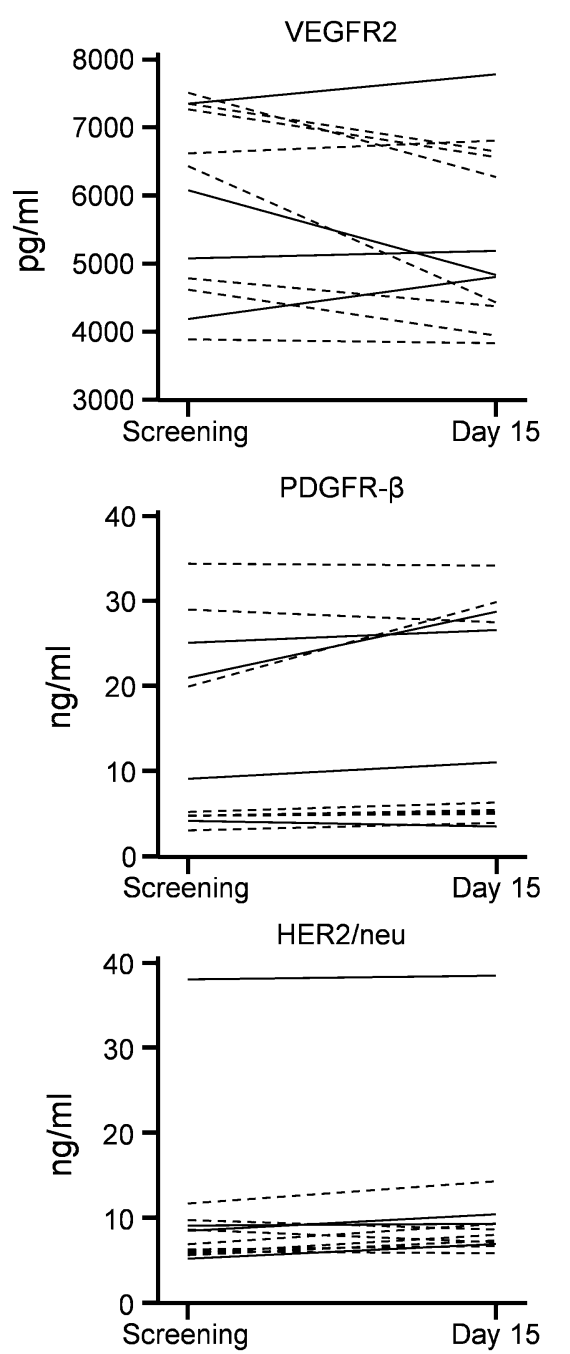

metastatic or advanced gastric cancer in a phase II trial (ECOG 5203) and demonstrated an encouraging efficacy profile with a median overall survival of 13.6 months [9]. These studies and our present trial support the use of sorafenib in combination with other chemotherapeutic agents against advanced gastric cancer.

The most common adverse events observed in this study were anorexia, rash/desquamation, neutropenia, thrombocytopenia, hand-foot reaction, nausea, leukopenia, fatigue, and elevation of lipase. All these adverse events were already reported in the SPIRITS trial (S-1 plus CDDP regimen for gastric cancer) or the TARGET study (sorafenib monotherapy for renal cell carcinoma). There was no specific or serious adverse event newly reported in this study, suggesting that the combination of the three drugs may cause no serious drug interaction. In terms of elevation of lipase, this was reported in TARGET study [15] as one of the most common laboratory abnormalities
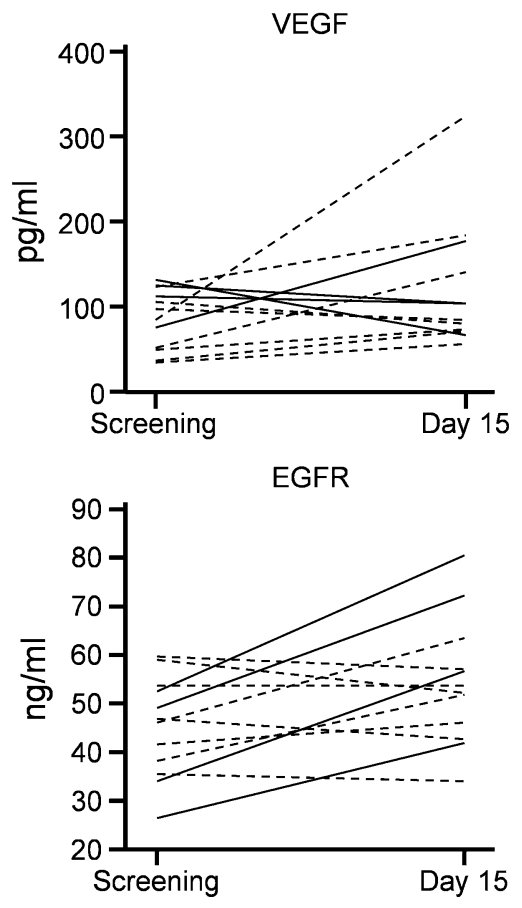

Best response:

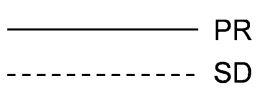

Fig. 5 Changes in plasma concentrations of various biomarkers [before treatment (screening) and on day 15]. VEGFR2 vascular endothelial growth factor receptor-2, PDGFR platelet-derived growth factor receptor, EGFR epidermal growth factor receptor 
Table 11 Summary of immunohistochemical staining (percent positive)

\begin{tabular}{|c|c|c|c|c|c|c|c|c|c|}
\hline \multirow[t]{2}{*}{ Patient ID } & \multirow[t]{2}{*}{$\mathrm{BR}$} & \multirow[t]{2}{*}{ Treatment } & \multirow{2}{*}{$\begin{array}{l}\text { VEGFR-2 } \\
\% \text { Positive }\end{array}$} & \multicolumn{6}{|c|}{$H$ score } \\
\hline & & & & AKT & $\begin{array}{l}\text { pAKT } \\
\text { (clone D9E) }\end{array}$ & $\begin{array}{l}\text { pAKT } \\
\text { (clone 14-5) }\end{array}$ & ERK & $\begin{array}{l}\text { pERK } \\
\text { (clone MAPK-YT) }\end{array}$ & $\begin{array}{l}\text { pERK } \\
\text { (clone D13.14.4E) }\end{array}$ \\
\hline \multirow[t]{2}{*}{ 20001-1008 } & PR & Screening & 20.22 & 5 & 0 & 23 & 0 & 10 & 5 \\
\hline & & End of $\mathrm{C} 2$ & & & & 30 & & & \\
\hline \multirow[t]{2}{*}{ 20003-1003 } & SD & Screening & & 1 & 100 & 72 & 265 & 1 & 1 \\
\hline & & End of $\mathrm{C} 2$ & 15.49 & 20 & 10 & 33 & 130 & 10 & 21 \\
\hline
\end{tabular}

Blank spaces indicate that no tumor was present and the percentage could not be evaluated

$B R$ best response

Table 12 Gene ontology classification of the top ten probes

\begin{tabular}{|c|c|c|c|c|c|}
\hline affy_id & $\begin{array}{l}\text { Gene } \\
\text { symbol }\end{array}$ & Gene name & Direction & $\begin{array}{l}\text { Indicative GO } \\
\text { classification } \\
\text { (component) }\end{array}$ & $\begin{array}{l}\text { Indicative GO } \\
\text { classification (function) }\end{array}$ \\
\hline 213953_at & KRT20 & Keratin 20 & Down & $\begin{array}{l}\text { Cytoskeleton part } \\
\text { Intermediate filament } \\
\text { cytoskeleton }\end{array}$ & $\begin{array}{l}\text { Structural constituent of } \\
\text { cytoskeleton }\end{array}$ \\
\hline 217564_s_at & CPS1 & $\begin{array}{l}\text { Carbamoyl-phosphate synthase } 1 \text {, } \\
\text { mitochondrial }\end{array}$ & Down & $\begin{array}{l}\text { Mitochondrial part } \\
\text { Mitochondrial inner } \\
\text { membrane }\end{array}$ & $\begin{array}{l}\text { Amino acid binding } \\
\text { Glutamate binding }\end{array}$ \\
\hline 209816_at & PTCH1 & Patched 1 & Down & $\begin{array}{l}\text { Plasma membrane } \\
\text { Integral to plasma } \\
\text { membrane }\end{array}$ & $\begin{array}{l}\text { Transmembrane receptor } \\
\text { activity } \\
\text { Signal transducer activity }\end{array}$ \\
\hline 243018_at & & Unidentified & Down & Not annotated & Not annotated \\
\hline 232315_at & ZNF880 & Zinc finger protein 880 & Up & Intracellular & $\begin{array}{l}\text { Zinc ion binding } \\
\text { Nucleic acid binding }\end{array}$ \\
\hline 213843_x_at & AC133561.1 & $\begin{array}{l}\text { Solute carrier family } 6 \text { (neurotransmitter } \\
\text { transporter, creatine), member } 8\end{array}$ & Down & Not annotated & Not annotated \\
\hline 37892_at & COL11A1 & Collagen, type XI, alpha 1 & Down & $\begin{array}{l}\text { Extracellular region part } \\
\text { Extracellular matrix part } \\
\text { Collagen }\end{array}$ & $\begin{array}{l}\text { Extracellular matrix } \\
\text { structural constituent } \\
\text { Structural molecule } \\
\text { activity }\end{array}$ \\
\hline 206828_at & TXK & TXK tyrosine kinase & Up & Cell part & $\begin{array}{l}\text { Protein tyrosine kinase } \\
\text { activity }\end{array}$ \\
\hline 234973_at & SLC38A5 & Solute carrier family 38 , member 5 & Up & $\begin{array}{l}\text { Integral to membrane } \\
\text { Plasma membrane }\end{array}$ & $\begin{array}{l}\text { Active transmembrane } \\
\text { transport activity } \\
\text { Amino acid } \\
\text { transmembrane transport } \\
\text { activity }\end{array}$ \\
\hline 214712_at & & Unidentified & Up & Not annotated & Not annotated \\
\hline
\end{tabular}

of grade 3 or 4 . Thus, toxicity observed in this study was consistent with the known side effects of S-1 plus CDDP combination therapy and sorafenib monotherapy. The frequency of common adverse events, however, tended to be higher in the present study than that in the SPIRITS trial and TARGET study.

In the present study, preliminary evaluations showed an encouraging efficacy profile, although the sample size of this study was small. Overall, 5 patients $(38.5 \%)$ showed partial response (PR) and the remaining $8(61.5 \%)$ showed stable disease (SD) whereas none of the patients showed progressive disease (PD). The ratio of PR in this study was lower than the SPIRITS trial [2]; however, the number of patients was relatively small for PK evaluation in the combination treatment, so it might be hard to compare this result with other studies and to obtain conclusions. It is noteworthy that all 12 patients having a target lesion for evaluation showed reduction of the target lesion after study treatment. 
The median RDI of sorafenib $(50.0 \%)$ was relatively lower than those of S-1 (89.3\%) and CDDP $(92.0 \%)$, and in median RDI from cycles 1 to 4 , RDI of sorafenib was decreasing gradually, whereas on the other hand those of S-1 and CDDP were sustained more than $80 \%$. One of the causes for this was the different dose reduction between these drugs $(50 \%$ reduction for sorafenib versus $20 \%$ for S-1 and $25 \%$ for CDDP at level 1). In terms of adverse events that eventually led to discontinuation permanently, namely, diarrhea, elevation of transaminase, myelosuppression, and skin toxicities (e.g., hand-foot skin reaction and rash), care should be taken.

In the present study, because of the negative results of the FLAGS study, a one-dose regimen based on the SPIRITS study was administered. In the future, therefore, considering these points such as dose reduction criteria in the protocol and adverse events that caused discontinuation in this study, efficacy should be confirmed in large-scale cohort studies using the combination regimen in this study.

The plasma concentration of 5-FU peaked at $4 \mathrm{~h}$ and gradually decreased thereafter both in patients who received S-1 alone (day 1) and in those who received the combination of three drugs (day 8) (Fig. 4). Geometric means of plasma 5-FU concentrations were slightly higher when 5-FU was administered in combination with two other drugs (day 8) than that when administered alone (day 1). In addition, mean ratios (day 8/day 1 ) of $C_{\max }$ and $\mathrm{AUC}_{0-\mathrm{tn}}$ of 5-FU were 1.64 and 1.56 , respectively, in 13 patients in whom these measurements were obtained on both day 1 and day 8 (Table 6).

In a previous pharmacokinetic study [16], the day 5/day 1 ratio of $C_{\max }$ and $\mathrm{AUC}_{0-10}$ of 5 -FU when $\mathrm{S}-1$ was administered alone for 5 days was $1.60\left(230 / 144 \mathrm{ng} \mathrm{ml}^{-1}\right)$ and $1.59\left(1364 / 857 \mathrm{ng} \mathrm{h} \mathrm{ml}^{-1}\right)$, respectively. This finding suggested that plasma 5-FU concentration increased to day 2 and achieved steady state after multiple administration of S-1. The observed ratios (day 5/day 1) in that study were similar to those (day 8/day 1) in our present study.

In our study, the influence of accumulation by repeated administration of S-1 cannot be ruled out as a cause of increased 5-FU exposures on day 8.

Among the biomarkers tested in this study, plasma concentration of EGFR tended to increase more during treatment in patients showing PR than in those showing $\mathrm{SD}$, although there was no difference in baseline plasma EGFR level between patients grouped by best response. Although the sample size here was small, it is interesting to speculate as to possible mechanisms of this phenomenon whose clinical relevance remains unknown. The ectodomain (ECD) of EGFR is detected in serum from patients with gastric cancer [17] and is thought to be shed from the cell surface via proteolytic cleavage and released into circulation. An in vitro study suggested that a disintegrin and metalloproteinase (ADAM) is involved in proteolytic release of ECD of EGFR [18]. Interestingly, it is also reported that colorectal cancer tumors responded to 5-FU treatment by activating ADAM17, which resulted in increased shedding of a EGFR ligand such as transforming growth factor (TGF)- $\alpha$ [19]. Simultaneous monitoring of soluble EGFR and its ligands such as EGF and TGF- $\alpha$ in plasma could provide further insights into clinical implication of change in plasma EGFR after chemotherapy treatment including 5-FU.

Results of histological and gene expression analysis should be interpreted cautiously because matched samples with tumors both before and after treatment were available only in limited patients. Although the samples for IHC analysis and gene expression analysis were collected during gastroscopy and therefore with visual inspection of the sampling sites, the fraction of tumor material was variable and about half of the samples for IHC analysis were found to consist of only normal gastric epithelium. Thus, differences detected between the pre- and post-treatment samples in gene expression analysis may also reflect differences between normal and tumor tissue as well as effects of therapy. Despite these caveats, the results from the GO classification seem to show a general trend in that the major expression changes were seen in genes required for maintaining tissue integrity and gastric epithelial function, rather than effects on genes involved in tumor pathogenesis.

In conclusion, the present phase I study demonstrates the acceptable toxicity and preliminary efficacy of combined treatment with S-1, CDDP, and sorafenib. Pharmacokinetic results suggested that combination of S- 1 and CDDP did not affect the PK of sorafenib. It is important to further investigate the mechanism underlying the efficacy of this combination therapy in a large-scale cohort study in the future.

Acknowledgments This study was funded by Bayer Yakuhin Ltd., Osaka, Japan. Hironobu Minami has received honoraria from Bayer Yakuhin Ltd., Osaka, Japan. Kensei Hashizume, Yoshihiro Kuroki, and Yuichiro Ito are employees of Bayer Yakuhin Ltd., Osaka, Japan. The other authors have no disclosures.

\section{References}

1. Jemal A, Bray F, Center MM, Ferlay J, Ward E, Forman D. Global cancer statistics. CA Cancer J Clin 2011;61:69-90.

2. Koizumi W, Narahara H, Hara T, et al. S-1 plus cisplatin versus S-1 alone for first-line treatment of advanced gastric cancer (SPIRITS trial): a phase III trial. Lancet Oncol. 2008;9:215-21.

3. Jin M, Lu H, Li J, et al. Randomized 3-armed phase III study of S-1 monotherapy versus S-1/CDDP (SP) versus 5-FU/CDDP (FP) in patients (patients) with advanced gastric cancer (AGC): SC-101 study. J Clin Oncol 2008;26:15s(supple;abstr4533)

4. Wilhelm SM, Carter C, Tang L, et al. BAY 43-9006 exhibits broad spectrum oral antitumor activity and targets the RAF/MEK/ ERK pathway and receptor tyrosine kinases involved in tumor progression and angiogenesis. Cancer Res. 2004;64:7099-109. 
5. Downward J. Targeting RAS signalling pathways in cancer therapy. Nat Rev Cancer. 2003;3:11-22.

6. McCubrey JA, Steelman LS, Chappell WH, et al. Roles of the Raf/MEK/ERK pathway in cell growth, malignant transformation and drug resistance. Biochim Biophys Acta. 2007;1773:1263-84.

7. Boku N, Ohtsu A, Nagashima F, Shirao K, Koizumi W. Relationship between expression of vascular endothelial growth factor in tumor tissue from gastric cancers and chemotherapy effects: comparison between S-1 alone and the combination of S-1 plus CDDP. Jpn J Clin Oncol. 2007;37:509-14.

8. Lieto E, Ferraraccio F, Orditura M, et al. Expression of vascular endothelial growth factor (VEGF) and epidermal growth factor receptor (EGFR) is an independent prognostic indicator of worse outcome in gastric cancer patients. Ann Surg Oncol. 2008;15:69-79.

9. Sun W, Powell M, O'Dwyer PJ, Catalano P, Ansari RH, Benson $\mathrm{AB}$ 3rd. Phase II study of sorafenib in combination with docetaxel and cisplatin in the treatment of metastatic or advanced gastric and gastroesophageal junction adenocarcinoma: ECOG 5203. J Clin Oncol. 2010;28:2947-51.

10. Ajani JA, Rodriguez W, Bodoky G, et al. Multicenter phase III comparison of cisplatin/S-1 with cisplatin/infusional fluorouracil in advanced gastric or gastroesophageal adenocarcinoma study: the FLAGS trial. J Clin Oncol. 2010;28:1547-53.

11. Kloft C, Appelius H, Siegert W, Schunack W, Jaehde U. Determination of platinum complexes in clinical samples by a rapid flameless atomic absorption spectrometry assay. Ther Drug Monit. 1999;21:631-7.

12. Gentleman RC, Carey VJ, Bates DM, et al. Bioconductor: open software development for computational biology and bioinformatics. Genome Biol. 2004;5:R80.
13. Boyle EI, Weng S, Gollub J, et al. GO: TermFinder-open source software for accessing gene ontology information and finding significantly enriched gene ontology terms associated with a list of genes. Bioinformatics (Oxford, England). 2004;20:3710-5.

14. Kim C, Lee JL, Choi YH, et al. Phase I dose-finding study of sorafenib in combination with capecitabine and cisplatin as a first-line treatment in patients with advanced gastric cancer. Invest New Drugs. 2012;30:306-15.

15. Escudier B, Eisen T, Stadler WM, et al. Sorafenib in advanced clear-cell renal-cell carcinoma. N Engl J Med. 2007;356:125-34.

16. Yamada Y, Hamaguchi T, Goto M, et al. Plasma concentrations of 5-fluorouracil and F-beta-alanine following oral administration of S-1, a dihydropyrimidine dehydrogenase inhibitory fluoropyrimidine, as compared with protracted venous infusion of 5-fluorouracil. Br J Cancer. 2003;89:816-20.

17. Choi JH, Oh JY, Ryu SK, et al. Detection of epidermal growth factor receptor in the serum of gastric carcinoma patients. Cancer (Phila). 1997;79:1879-83.

18. Sanderson MP, Keller S, Alonso A, Riedle S, Dempsey PJ, Altevogt P. Generation of novel, secreted epidermal growth factor receptor (EGFR/ErbB1) isoforms via metalloprotease-dependent ectodomain shedding and exosome secretion. J Cell Biochem. 2008;103:1783-97.

19. Kyula JN, Van Schaeybroeck S, Doherty J, Fenning CS, Longley DB, Johnston PG. Chemotherapy-induced activation of ADAM17: a novel mechanism of drug resistance in colorectal cancer. Clin Cancer Res. 2010;16:3378-89. 